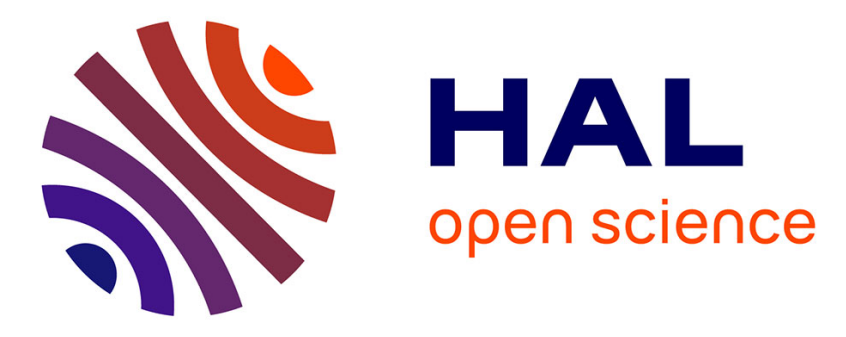

\title{
Ultraviolet Glow from the Hydrogen Wall
}

Eric Quémerais, Rosine Lallement, Jean-Loup Bertaux, B. R. Sandel, V.

Izmodenov, Y. Malama

\section{To cite this version:}

Eric Quémerais, Rosine Lallement, Jean-Loup Bertaux, B. R. Sandel, V. Izmodenov, et al.. Ultraviolet Glow from the Hydrogen Wall. The Astrophysical Journal, 2010, 711 (2), pp.1257-1262. 10.1088/0004-637X/711/2/1257 . hal-00460874

\section{HAL Id: hal-00460874 https://hal.science/hal-00460874}

Submitted on 7 Jul 2020

HAL is a multi-disciplinary open access archive for the deposit and dissemination of scientific research documents, whether they are published or not. The documents may come from teaching and research institutions in France or abroad, or from public or private research centers.
L'archive ouverte pluridisciplinaire HAL, est destinée au dépôt et à la diffusion de documents scientifiques de niveau recherche, publiés ou non, émanant des établissements d'enseignement et de recherche français ou étrangers, des laboratoires publics ou privés. 


\title{
ULTRAVIOLET GLOW FROM THE HYDROGEN WALL
}

\author{
E. Quémerais ${ }^{1}$, R. Lallement ${ }^{1}$, J. L. Bertaux ${ }^{1}$, B. R. Sandel ${ }^{2}$, V. Izmodenov ${ }^{3}$, and Y. Malama ${ }^{3}$ \\ ${ }^{1}$ Université Versailles-St Quentin, LATMOS-IPSL, Verrières le Buisson, France; eric.quemerais@latmos.ipsl.fr \\ ${ }^{2}$ Lunar and Planetary Laboratory, University of Arizona, Tucson, Az, USA \\ ${ }^{3}$ Institute for Problems in Mechanics, Moscow, Russia \\ Received 2009 August 28; accepted 2010 January 21; published 2010 February 23
}

\begin{abstract}
We compare new results of models of the interplanetary H Ly $\alpha$ intensity background in the outer heliosphere with scans performed by the Voyager 1/2 UV spectrometer (UVS) instruments between 1993 and 2003. This study shows that the excess intensity initially reported by Quémerais et al. can be explained by models of the hydrogen atom distribution including effects of the heliospheric interface. The models of the hydrogen atom distribution in the interplanetary medium used in this work have been developed following the numerical scheme presented by Baranov \& Malama. Recent improvements are described by Izmodenov et al. Radiative transfer computations of the interplanetary Ly $\alpha$ intensity are made following a Monte Carlo approach presented by Quémerais and Quémerais \& Izmodenov. We find that the upwind intensity excess observed in the outer heliosphere initially reported by Quémerais et al. can be explained by a full radiative transfer computation. This computation must include a full description of the velocity distributions of the different hydrogen populations that enter the heliosphere after crossing the interface. The excess upwind intensity observed by UVS on Voyager 1 and Voyager 2 can be explained as an emission of the decelerated hydrogen atoms near the stagnation point of the heliopause. Because those atoms are slowed down relative to the main hydrogen flow, photons they scatter suffer less absorption and are visible at a much larger distance than is the case for photons scattered by atoms in the main flow. The shape and extent of the excess emission gives information about the decelerated population near the heliopause stagnation point. A detailed comparison between the data and our present model does not show a complete agreement. The modeled intensity excess is larger than the observed one. We discuss possible improvements to the $\mathrm{H}$ distribution model in order to decrease the size of the excess in the model, for example, by decreasing the density of $\mathrm{H}$ atoms in the hydrogen wall.
\end{abstract}

Key words: interplanetary medium - ultraviolet: general

Online-only material: color figures

\section{INTRODUCTION}

The heliospheric interface is the region of space where the expanding solar wind interacts with the ionized component of the interstellar medium. This region constitutes the actual limit of the solar system. In recent years, the two Voyager spacecraft reached the doorstep of the heliospheric interface by crossing the termination shock of the solar wind (Stone et al. 2005). Although both spacecraft are still inside the inner heliosheath, where the decelerated solar wind is deflected, they are approaching the heliopause that separates the solar wind plasma from the ionized component of the interstellar medium. The neutral component of the interstellar medium crosses the heliospheric interface unhindered and has been observed in the inner heliosphere for decades (Bertaux \& Blamont 1971; Thomas \& Krassa 1971). This is called the interstellar neutral wind, and is mainly composed of hydrogen atoms. In the early 1990's, pioneering theoretical work by Baranov (1990) and Baranov \& Malama (1993) has demonstrated, however, that a large fraction of the neutral atoms are strongly affected when crossing the heliospheric interface, as previously suggested by Wallis (1974). The complex magnetic structure of the heliospheric interface acts directly only on charged particles. Yet, through the mechanism of resonant charge exchange, hydrogen atoms are strongly coupled to the ions. This results in deceleration and heating of the neutral hydrogen flow. Baranov \& Malama (1993) demonstrated that, because of this deceleration process through coupling by charge exchange, hydrogen atoms tend to pile up in the region called the outer heliosheath, where the interstellar plasma is deflected before reaching the interface. The pile-up is even more prominent near the stagnation point of the plasma flow. This is called the hydrogen wall. In 1995, by studying observations of nearby stars made by the Hubble Space Telescope in the far-UV, Linsky \& Wood (1995) showed that the hydrogen wall creates an absorption feature on the Ly $\alpha$ line of some stars. This constitutes the first detection of the hydrogen wall.

Here, we present new model computations of the interplanetary background intensity in the outer heliosphere. Our results show that the hydrogen wall backscatters solar Ly $\alpha$ photons and creates a small excess in the upwind direction. Because of the Doppler shift between the hydrogen wall atoms and the main flow of hydrogen in the heliosphere, these backscattered photons are visible from a much larger distance than the normal interplanetary glow. We argue that the UV spectrometers (UVS) on both Voyager spacecraft have observed this glow from the hydrogen wall as early as the early 1990's. At that time, the source of this emission was not identified because we lacked an accurate model of radiative transfer including the actual velocity distribution of hydrogen atoms. A detailed comparison between data and model still shows some disagreement. We discuss possible improvements to the model.

\section{THE INTERPLANETARY INTENSITY BACKGROUND}

\subsection{Complete Frequency Redistribution}

The interplanetary ultraviolet background is due to the backscatter of solar photons by atoms present in the interplane- 
tary medium. Its main component is the backscatter of solar $\mathrm{H}$ Ly $\alpha$ photons $(121.6 \mathrm{~nm})$ by hydrogen atoms in the interplanetary medium. This emission was first identified in the late 1960's by two experiments on the $O G O-5$ satellite (Bertaux \& Blamont 1971; Thomas \& Krassa 1971). Models of the hydrogen atom distributions in the inner heliosphere were developed in the early 1970's (Blum \& Fahr 1970). Studies of the interplanetary background have been used to infer parameters of the insterstellar wind flow and even the direction of the interstellar magnetic field (Lallement et al. 2005). The UVS instruments aboard Voyager 1 and Voyager 2 have observed the interplanetary background since the beginning of the spacecraft cruise to the outer planets and have performed many observations after the last planetary encounter. In 2009, the UVS instrument on-board Voyager 1 is still making observations of the interplanetary background.

Both Voyager spacecraft are traveling toward directions that are close to the upwind direction. This is the direction from which the interstellar wind appears to come in the solar rest frame, due to the relative velocities of the solar system and the local interstellar cloud. In 1993, dedicated observations started to measure the intensity ratio from the upwind to the downwind directions (Quémerais et al. 1995). The upwind direction pointed toward the hydrogen wall and the downwind direction close to the Sun and the inner heliosphere. It was then found that the backscattered emission in the outer heliosphere shows a small excess close to the upwind direction when compared to a classical hot model (Quémerais et al. 1995). To investigate the origin of this excess emission, Quémerais et al. (1996) compared the data with model computations based on the hydrogen atom distributions derived from the model of Baranov \& Malama (1993). Radiative transfer computations were done using some simplifying assumptions. The radiative transfer model assumed an axisymmetric geometry for the hydrogen distribution. Number density values were used including the large increase at the position of the hydrogen wall. This was the main difference from the classical hot model which assumes that the hydrogen number density is constant at large distance from the Sun (outside $50 \mathrm{AU}$ ). It was also assumed that the hydrogen flow is isothermal with a constant bulk velocity. Finally, the scattering process was represented through the assumption of complete frequency redistribution. This assumption is commonly used for modeling planetary exospheres. The works of Keller et al. (1981), Hall et al. (1993), and Quémerais \& Bertaux (1993) used the hypothesis of complete frequency redistribution which assumes that there is no link between the frequencies of the photon before and after scattering. In that case, the emission profile at one point is simply defined by the local temperature of the hydrogen distribution and is independent of the incoming profile.

Figure 1 shows a map of the interplanetary background computed for an observer at $60 \mathrm{AU}$ from the Sun in the upwind direction. The model uses the simplifying assumptions described above. Although the hydrogen number density follows the values of Baranov \& Malama (1993), the hydrogen wall is not visible because the medium is optically thick at Ly $\alpha$. For such a model, the main factor is the optical thickness between the source of photons (the Sun) and the scattering point. In that case, the upwind direction is always dimmer. Note that this does not mean that a hot model computation and an interface model computation give the same result. The difference between the two types of hydrogen distribution appears in the upwind to downwind intensity ratio, which is modulated by the hydrogen density gradient at the position of the observer. Indeed, the

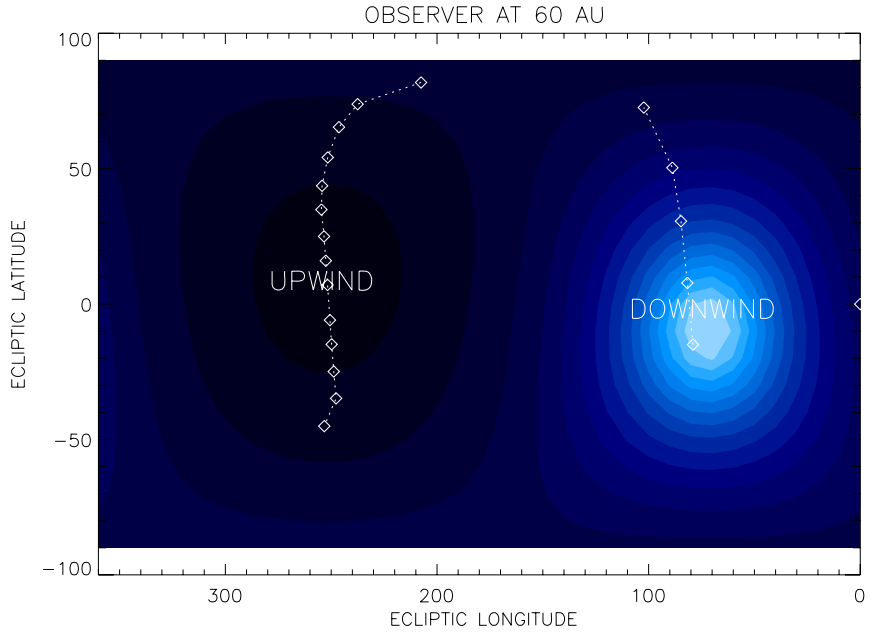

Figure 1. Model map of the interplanetary background computed for an observer at $60 \mathrm{AU}$. The observer's position is along the upwind axis, so there is no parallax with the upwind direction. The hydrogen number density values are derived from the model of Baranov \& Malama (1993). The gas is assumed isothermal, with a constant bulk velocity of $22 \mathrm{~km} \mathrm{~s}^{-1}$ in the solar rest frame. The diamonds show the pointing directions of the UVS scans from downwind to upwind. The color code is arbitrary and depends on model parameters, with lighter colors for brighter regions.

(A color version of this figure is available in the online journal.)

measured ratio demonstrated the existence of the interface, that is, a steeper density increases from the inner to the outer heliosphere as compared to the classical cavity without interface. But, for both models, the upwind direction always shows a shallow minimum in intensity. This result was pointed out by Quémerais et al. (1996) on the basis of the models available at the time. These authors concluded that the observed excess in the Voyager UVS data could not come from the hydrogen wall. In the model, the hydrogen wall was too far away from the Voyager spacecraft to be the source of the excess glow. It was shown that a distance of $10 \mathrm{AU}$ roughly corresponds to an optical thickness at line center of 1 , i.e., $10 \mathrm{AU}$ is the mean distance traveled by a photon at line center before the next scattering. With a heliopause more than $50 \mathrm{AU}$ away from the spacecraft, photons scattered at the wall could not be seen directly. No model with reasonable parameters could actually produce a local maximum in the upwind direction.

Quémerais et al. (1996) concluded that the upwind to downwind intensity ratio was strongly affected by the change in distribution in the outer heliosphere caused by the hydrogen wall, but that the local maximum in the upwind direction was not caused by the wall itself. Various alternative explanations were proposed, including a small residual emission due to recombination in H II regions close to the galactic plane which lies within 20 degrees of the upwind direction. The galactic longitude intervals for the excess roughly correspond to the Sagittarius spiral arm (Lallement 1996).

\subsection{Angle Dependent Partial Frequency Redistribution}

Following this preliminary analysis, the models were substantially improved, first by defining more accurately the hydrogen atom distributions (Izmodenov et al. 2001), and second by computing line profiles in the interplanetary medium (Quémerais 2000; Quémerais \& Izmodenov 2002). To achieve this second goal, we replaced the initial assumption of Complete Frequency Redistribution by the exact formulation given by the Angle Dependent Partial Frequency Redistribution (ADPFR; 
Mihalas 1970). This function expresses the energy of the scattered photon as a function of the velocity vector of the atom, the energy of the initial photon, and the scattering angle. This results in the following cases.

1. The absorption profile in the local rest frame of the computation cell is proportional to a normal Gaussian profile with a temperature equal to the temperature of the local velocity distribution projected on the incident direction.

2. If the emergent direction is perpendicular to the incident direction, the scattered profile is a Gaussian profile (Complete Frequency Redistribution).

3. If the emergent and incident directions are colinear, the scattered profile is proportional to the incident profile multiplied by the cross section profile (Coherent Scattering).

4. For other scattering angles, the emitted profile is the sum of a coherent profile and a Gaussian, the temperature of which is defined by the direction perpendicular to the incident direction and contained inside the plane defined by both emergent and incident directions (angle-dependent redistribution).

Accurate velocity distributions of hydrogen atoms were also used. These distributions are described in a review by Izmodenov et al. (2009). The models are kinetic-gas dynamic representations of the heliospheric interface between the solar wind and the local interstellar medium. In the distributions, we used below, the interstellar magnetic field was set to zero. However, we expect some effects of the interstellar magnetic field on the UVS maps. This will be studied in detail in future works. Early computations have shown that the magnetic field does not remove the hydrogen wall but mainly displaces it. The models assumed that the interstellar gas temperature is $6500 \mathrm{~K}$, the relative velocity with the Sun is $26 \mathrm{~km} \mathrm{~s}^{-1}$. The proton density is $0.04 \mathrm{~cm}^{-3}$ and the hydrogen density is $0.18 \mathrm{~cm}^{-3}$, before filtration by the interface. This allowed us to compute line profiles seen by an observer anywhere in the heliosphere. Using these improved models, we have reanalyzed the results of Quémerais et al. (1996).

An example of these new computations is shown in Figure 2. We have computed the interplanetary background map for the same geometry as in Figure 1. But in this new case, we use the full distribution of hydrogen atom velocities and not only hydrogen number densities. Multiple scattering effects are computed using the ADPFR representation of the scattering process. The hydrogen distribution in the heliosphere is described by Izmodenov et al. (2001). The hydrogen atoms are divided into four different populations. The populations are numbered according to their source region. Population 1 includes $\mathrm{H}$ atoms created after charge exchange with protons of the supersonic solar wind. With velocities larger than $300 \mathrm{~km} \mathrm{~s}^{-1}$, these atoms are Doppler-shifted outside of the solar Ly $\alpha$ illuminating line. They do not contribute to the interplanetary glow. Population 2 is created in the inner heliosheath where the solar wind is subsonic. The resulting hot population contributes only marginally to the interplanetary background. The main contributors to the interplanetary background are populations 3 and 4 . The $\mathrm{H}$ atoms from population 3 are created by charge exchange with protons of the decelerated interstellar plasma. These atoms are decelerated near the heliopause and accumulate, thus forming the hydrogen wall. Finally, population 4 includes $\mathrm{H}$ atoms which cross the interface without charge exchange. The velocity distribution of population 4 is slightly cooler and faster than the distribution of $\mathrm{H}$ atoms in the interstellar medium because the

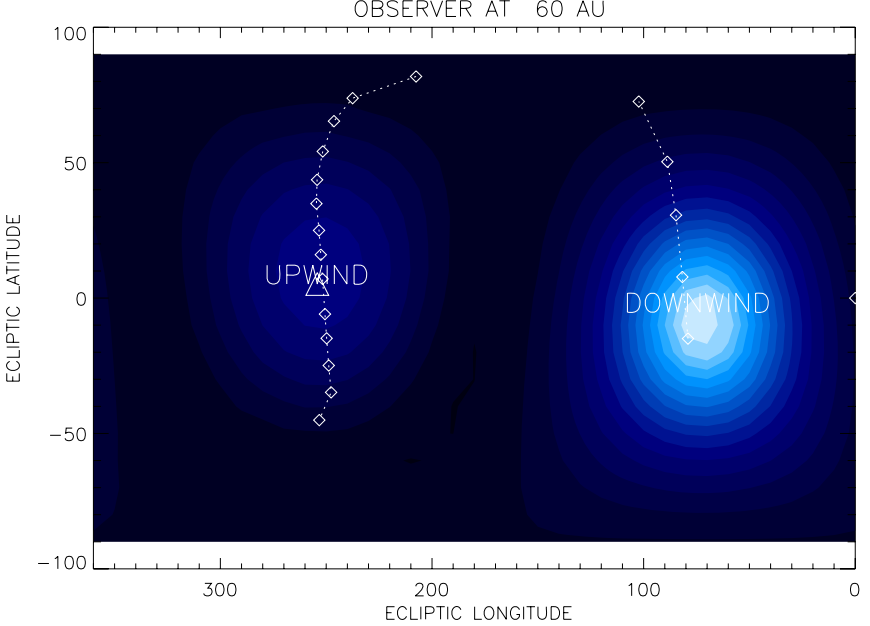

Figure 2. Model of the interplanetary background as seen from $60 \mathrm{AU}$ in the upwind direction. The observer's position is along the upwind axis, so there is no parallax with the upwind direction. This model computation uses complete distributions of the hydrogen atoms, number density, velocity, and temperature. The local maximum in the upwind direction is created by backscattered photons from the decelerated population of hydrogen atoms at the hydrogen wall. The diamonds show the line-of-sight directions of the observations of the Voyager 1 UVS scans. The color code is arbitrary and depends on model parameters, with lighter colors for brighter regions.

(A color version of this figure is available in the online journal.)

slower atoms have a larger probability to charge exchange than the faster ones. The map in Figure 2 is computed using populations 2,3 , and 4 . The intensity backscattered by population 2 is equal to a few rayleigh. For comparison, the intensity backscattered by populations 3 and 4 is higher by 2 orders of magnitude.

The most striking difference between the two models appears in Figure 2 where a secondary maximum is present in the upwind direction in the case of the ADPFR model. The primary maximum is in the direction of the Sun which is the source of the photons. This secondary bump is caused by the hydrogen wall, or more precisely by the slow hydrogen atoms in the hydrogen wall (population 3). These atoms, created after charge exchange with the decelerated interstellar plasma close to the stagnation point of the heliopause, have a bulk velocity in the solar rest frame close to zero, i.e., the velocity of the plasma before charge exchange. This means that these atoms are shifted from the bulk flow which has a mean velocity of $22 \mathrm{~km} \mathrm{~s}^{-1}$. Considering that a temperature of $10^{4} \mathrm{~K}$ corresponds to a thermal width of $13 \mathrm{~km} \mathrm{~s}^{-1}$ in the velocity distribution, we see that these decelerated atoms are significantly Doppler-shifted from the rest of the hydrogen atoms between them and the Sun. As a consequence, they scatter photons that are beyond the wings of the absorption profile in the interplanetary medium. Finally, because the Voyager spacecraft are close to the wind axis, the scattering is almost coherent (scattering angle close to $180^{\circ}$ ). This means that a photon scattered by an atom at the edge of the wing of the main velocity profile stays in the wing of the absorption profile of the main flow. Because the optical thickness in the wing is much smaller than the value at the line center, photons scattered at the hydrogen wall can be detected at a much larger distance. This explains the secondary maximum in intensity seen in Figure 2 in the upwind direction. The model shown in Figure 1 does not have a bump because it does not account for the velocity difference between the hydrogen wall and the flow in the heliosphere. 


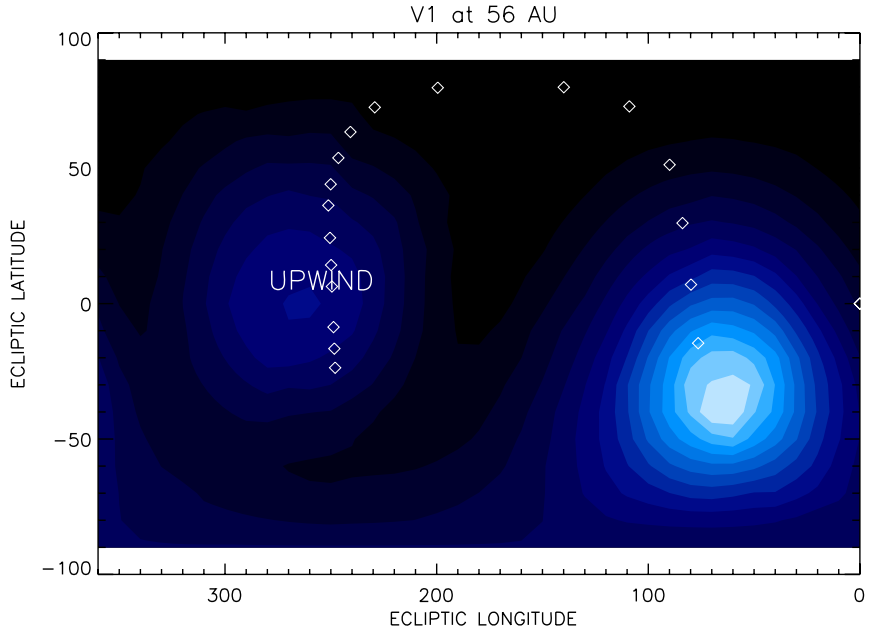

Figure 3. Model of the interplanetary map computed for the actual position of Voyager 1 at $56 \mathrm{AU}$ from the Sun. In the upwind direction (closest to point 14), we see a local maximum in the model. The parallax effect tends to shift the positions of maxima toward lower ecliptic latitudes because Voyager 1 is above the ecliptic plane. The diamonds show the line-of-sight directions of the observations of the Voyager 1 UVS scans. The last scan was performed in 2003. The color code is arbitrary and depends on model parameters, with lighter colors for brighter regions.

(A color version of this figure is available in the online journal.)

\section{DATA AND MODEL COMPARISON}

\subsection{Outer Heliosphere Scans}

The UVS instruments of Voyager 1 and Voyager 2 have performed many scans in the outer heliosphere. These data are described in detail in Quémerais et al. (2003, 2009). The excess intensity in the Ly $\alpha$ maps is shown in detail in Quémerais et al. (1995). It was still present in the last outer heliosphere scan performed by Voyager 1 UVS in 2003, which was stopped for lack of power to activate the scan platform.

Figure 3 shows the model map of the Ly $\alpha$ background obtained for Voyager 1 UVS at 56 AU. The main difference between Figures 2 and 3 is that, in the second computation, we have used the actual position of the spacecraft, whereas in Figure 2 the observer sits on the upwind axis from the Sun. Therefore, in Figure 3, we see a parallax shift of the position of the downwind maximum. Since Voyager 1 is above the ecliptic plane, the direction to the Sun is moved toward lower latitudes. Similarly, the secondary maximum is also shifted due to parallax toward lower latitudes but to a lesser extent. In Figure 3, we have overplotted the directions of the lines of sight of the Voyager 1 outer heliosphere scans (diamonds). The directions of the data points are also shown in Figures 1 and 2 as diamonds. The reference angle is the downwind direction (point 1). The points are numbered from 1 to 18 . The spacing between points is roughly $10^{\circ}$ in the upwind hemisphere and $20^{\circ}$ in the downwind hemisphere. The upwind direction is closest to points 13 and 14. We see that the scans were designed to start close to the maximum of intensity in the downwind direction and roughly follow a great circle passing through the upwind direction.

Figure 4 shows a similar computation made for the actual position of Voyager 2 at $44 \mathrm{AU}$ from the Sun. In that case, because Voyager 2 is below the ecliptic plane, the parallax shift moves the two maxima toward higher ecliptic latitudes. We have also overplotted the direction of sight of the Voyager 2 heliospheric scans (the last one was performed in 1998).

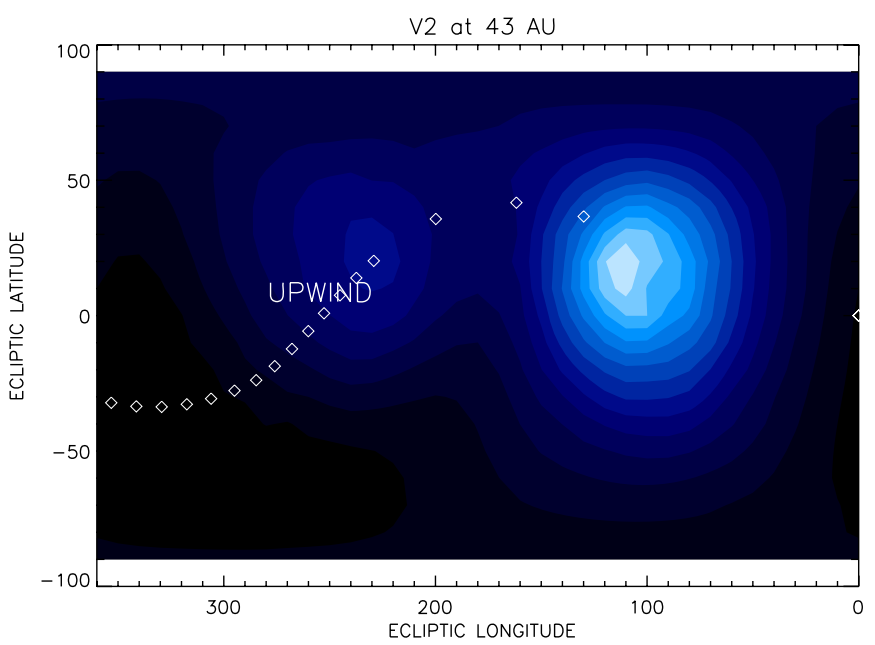

Figure 4. Model of the interplanetary map computed for the actual position of Voyager 2 at $44 \mathrm{AU}$ from the Sun. In the upwind direction (closest to point 6), we see a local maximum in the model. The parallax effect tends to shift the positions of two maxima towards higher ecliptic latitudes because Voyager 2 is below the ecliptic plane. The diamonds show the line of sight directions of the observations of the Voyager 2 UVS scans. The last scan was performed in 1998. (A color version of this figure is available in the online journal.)

\subsection{Data Model Comparisons}

The data of Ly $\alpha$ intensity values are shown in Figures 5 and 6. Details are given in Quémerais et al. (1995, 2003). Both the data and model of backscattered intensity clearly show a bump in the upwind direction. We are quite convinced that only a model incorporating a velocity difference between populations 3 and 4 will show such an excess. Indeed, it is caused by the fact that the solar line is wider than the Doppler width of the heliospheric hydrogen population. Therefore, solar photons can reach the atoms of population 3 at the location of the hydrogen wall and are scattered there. This will not happen if the velocity difference between populations 3 and 4 is smaller than the Doppler width of population 4. However, there is still a noticeable difference between the data and our model results. The model values show an excess which is significantly larger than the actual data.

Figure 5 shows the data (diamonds) recorded by Voyager 1/ UVS during an outer heliosphere scan. The values correspond to the points shown in Figure 3. We have overplotted the model results for the full computation (thick line) and for the test case with an isothermal flow with constant bulk velocity (dashed line). In the downwind direction (close to the Sun), we have scaled models and data to match one another. In the upwind direction, the CFR model shows a minimum, whereas both data and full model show an excess over the CFR model. Similarly, Figure 6 shows the results for Voyager 2 at 44 AU from the Sun. The corresponding map is shown in Figure 4. There again, the dashed line corresponds to the CFR model and shows a minimum in the upwind direction. The data show a small excess over this minimum in the upwind direction. The full model shows a significantly larger excess and this secondary bump seems shifted by parallax, more than the data actually show.

These computations give a strong indication that the numerical models of the hydrogen populations near the heliopheric interface have to be improved to account for these UVS observations. In the following section, we will consider a few possible ways to improve the results of this data and model comparison. 


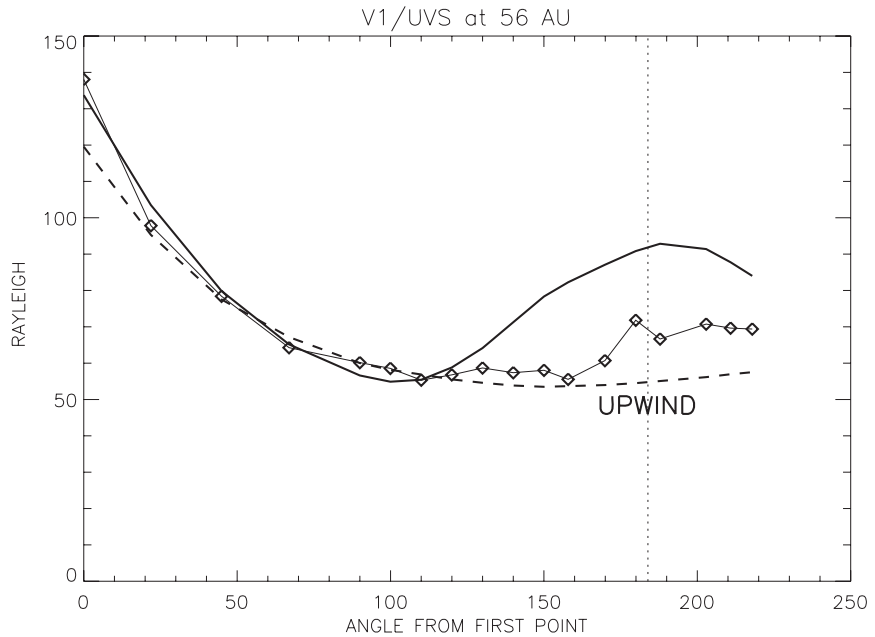

Figure 5. Model and data comparison for Voyager 1 at $56 \mathrm{AU}$ from the Sun. The abscissa gives the angle computed from the first direction which is close to downwind. The diamonds are the data points. The thick line is the full radiative transfer model computation. The dashed line shows the model result when the velocity shift of the hydrogen wall is not taken into account (CFR). The downwind values (direction close to the Sun) are scaled to the same values because this part of the heliosphere is less affected by the heliospheric interface. In the upwind direction (closest to points 13 and 14, shown by vertical dot line), we see a local minimum in the CFR model (dashed line). The complete model computation shows a large excess in this direction. The data show a smaller but significant excess over the CFR model in the upwind direction.

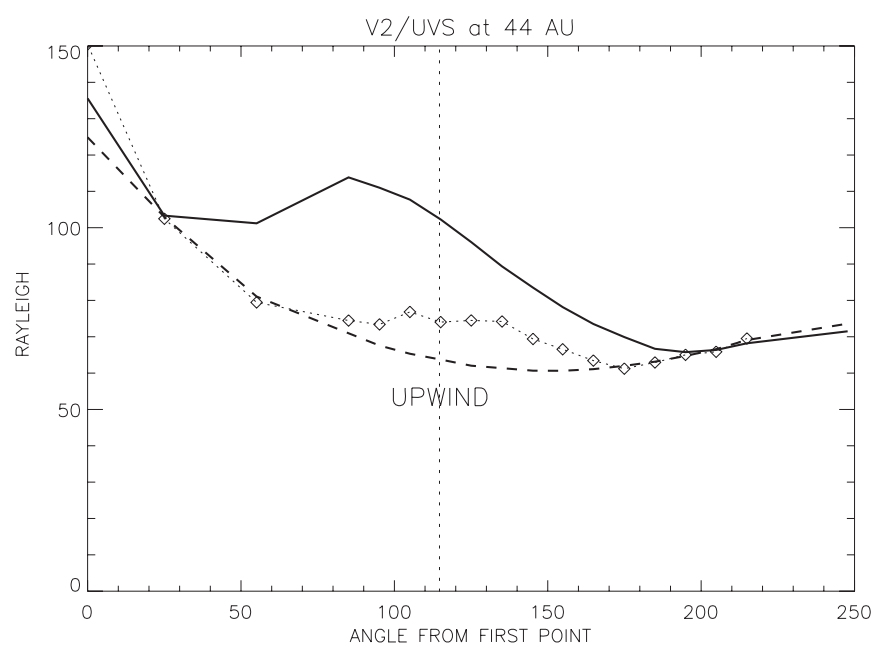

Figure 6. Model and data comparison for Voyager 2 at $44 \mathrm{AU}$ from the Sun. The axes are the same as in the previous figures. The diamonds are the data points. The thick line is the full radiative transfer model computation. The dashed line shows the model result when the velocity shift of the hydrogen wall is not taken into account (CFR). The values are for the diamonds shown in Figure 4. The downwind values (direction close to the Sun) are scaled to the same values. In the upwind direction (closest to point 7 , shown by vertical dot line), we see a local minimum in the CFR model (dashed line). As in Figure 5, the complete model computation shows a larger excess in this direction. The data show a smaller excess over the CFR model in the upwind direction.

\section{CONCLUSION}

New model computations have shown that the excess intensity observed by the Voyager UVS in the upwind direction (Quémerais et al. 1995) is due to the backscattering of solar photons by the slowed down hydrogen population trapped in the hydrogen wall. Because these photons are Doppler-shifted outside the absorption profile of the main flow, they are visible at a much larger distance than the usual $10 \mathrm{AU}$.
Therefore, the Voyager UVS data in the outer heliosphere give a direct observation of the hydrogen wall, mainly of the decelerated and heated population beyond the heliopause.

Interplanetary $\mathrm{Ly} \alpha$ intensity data and model comparisons still show a clear discrepancy on the position of this excess. The model intensity excess is also significantly larger than the observed one. Moreover, the model predicts a parallax shift of the position of the secondary maximum in the upwind direction. This parallax shift does not clearly appear in the data.

This is an indicator that the detailed structure of the interface and its effect on the hydrogen population need to be improved in our model.

Here, we suggest a few ways to improve the fit of the model to the data. Some of these changes may not be consistent with other aspects of the heliospheric interface structure. The simplest way to improve the model fit is to decrease the number density of population 3 . This would mean that less interstellar hydrogen atoms suffer charge exchange when crossing the interface region. In parallel, the model excess will be smaller if the velocity shift between populations 3 and 4 is smaller. This again will be true on average if fewer $\mathrm{H}$ atoms have a charge exchange at the interface. This solution will not change the lack of parallax effect observed in the data. To explain the lack of parallax effect, we can suggest that the population 3 is actually farther away from the spacecraft than what the model assumes. Since the location of the heliopause is rather well constrained, this could mean that the hydrogen wall is actually thicker than in the model distribution. This may be obtained by removing the bow shock in the interstellar medium. In the presence of a strong magnetic field, the interstellar gas flow is not superalfvenic and there is no bow shock. In that case, the deflection of the interstellar plasma may be spread over larger distances than in the case of the two-shock model. Population 3 would appear over greater distances thus creating a larger, and less dense, hydrogen wall.

These speculations will have to be checked in future background intensity model computations and comparisons with the Voyager Ly $\alpha$ UVS data.

V.I. was supported in part by President grant MD-3890.2009.2 and Dynastia Foundation. Y.M. was supported in part by RFBR grants 06-02-72557, and 07-02-01101, and Rosnauka contract 02.740 .11 .5025 .

\section{REFERENCES}

Baranov, V. B. 1990, Space Sci. Rev., 52, 89

Baranov, V. B., \& Malama, Y. G. 1993, J. Geophys. Res., 98, 15157

Bertaux, J. L., \& Blamont, J. E. 1971, A\&A, 11, 200

Blum, P. W., \& Fahr, H. J. 1970, Astrophys. Lett., 6, 127

Hall, D. T., Shemansky, D. E., Judge, D. L., Gangopadhyay, P., \& Gruntman, M. A. 1993, J. Geophys. Res., 98, 15,185

Izmodenov, V. V., Gruntman, M., \& Malama, Y. G. 2001, J. Geophys. Res., 106, 10681

Izmodenov, V., Malama, Y., \& Ruderman, M. S. 2005, A\&A, 429, 1069

Izmodenov, V. V., Malama, Y. G., Ruderman, M. S., Chalov, S. V., Alexashov, D. B., Katushkina, O. A., \& Provornikova, E. A. 2009, Space Sci. Rev., 146, 329

Keller, H. U., Richter, K., \& Thomas, G. E. 1981, A\&A, 102, 415

Lallement, R. 1996, Space Sci. Rev., 78, 361

Lallement, R., Quémerais, E., Bertaux, J. L., Ferron, S., Koutroumpa, D., \& Pellinen, R. 2005, Science, 307, 1447

Linsky, J. L., \& Wood, B. 1995, BAAS, 27, 1347

Mihalas, D. 1970, Astronomy and Astrophysics (Stellar Atmospheres Series of Books; San Francisco, CA: Freeman)

Quémerais, E. 2000, A\&A, 358, 353 
Quémerais, E., \& Bertaux, J. L. 1993, Adv. Space Res., 13, 298

Quémerais, E., Bertaux, J. L., Lallement, R., Sandel, B. R., \& Izmodenov, V. 2003, J. Geophys. Res., 108, 8029

Quémerais, E., Lallement, R., Sandel, B. R., \& Clarke, J. T. 2009, Space Sci. Rev., 143, 151

Quémerais, E., Malama, Y. G., Sandel, B. R., Lallement, R., Bertaux, J.-L., \& Baranov, V. B. 1996, A\&A, 308, 279
Quémerais, E., \& Izmodenov, V. 2002, A\&A, 396, 269

Quémerais, E., Sandel, B. R., Lallement, R., \& Bertaux, J.-L. 1995, A\&A, 299, 249

Stone, E. C., Cummings, A. C., McDonald, F. B., Heikkila, B. C., Lal, N., \& Webber, W. R. 2005, Science, 309, 2017

Thomas, G. E., \& Krassa, R. F. 1971, A\&A, 11, 218

Wallis, M. K. 1974, MNRAS, 167, 103 